TRANSACTIONS OF THE

AMERICAN MATHEMATICAL SOCIETY

Volume 359, Number 5, May 2007, Pages 2123-2136

S 0002-9947(06)04099-2

Article electronically published on December 15, 2006

\title{
INHOMOGENEOUS STRICHARTZ ESTIMATES FOR THE SCHRÖDINGER EQUATION
}

\author{
M. C. VILELA
}

\begin{abstract}
We study Strichartz estimates for the solution of the Cauchy problem associated with the inhomogeneous free Schrödinger equation in the case when the inital data is equal to zero, proving some new estimates for certain exponents and giving counterexamples for some others.
\end{abstract}

\section{INTRODUCTION}

Consider the Cauchy problem for the inhomogeneous free Schrödinger equation

$$
\left\{\begin{array}{l}
i \partial_{t} u+\Delta_{x} u=F(x, t), \quad(x, t) \in \mathbb{R}^{n} \times \mathbb{R}, \\
u(x, 0)=f(x) .
\end{array}\right.
$$

R. Strichartz (see 11]), following previous works of E. Stein, P. Tomas and I.E. Segal (see [10, [13], [14] and 9]), proved in 1977 the following inequality:

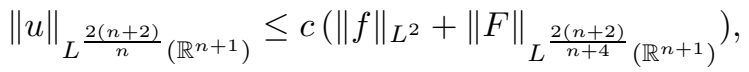

where $u$ is the solution of (11). After that, J. Ginibre and G. Velo, K. Yajima, T. Cazenave and F. Weissler, S.J. Montgomery-Smith, and M. Keel and T. Tao studied this estimate for more general spaces with different exponents in space and time (see [5], [15], 2], 8], [3, 7] and [12]).

It is well known that the solution of (11) can be written in the form

$$
u(x, t)=e^{i t \Delta} u_{0}(x)-i \int_{0}^{t} e^{i(t-\tau) \Delta} F(\cdot, \tau)(x) d \tau,
$$

where the first term is the solution of the homogeneous problem, that is, $F=0$ in (11), and the second one is the solution of the inhomogeneous problem with inital data $f=0$. The problem of determining in which Lebesgue spaces the solution of the homogeneous problem lies is completely solved, and we summarize it in the next theorem.

\section{Theorem 1.1.}

$$
\left\|e^{i t \Delta} f\right\|_{L_{t}^{q} L_{x}^{r}} \leq c\|f\|_{L^{2}},
$$

if and only if $q, r \geq 2,(q, r, n) \neq(2, \infty, 2)$ and $2 / q+n / r=n / 2$.

Received by the editors December 12, 2003 and, in revised form, March 1, 2005.

2000 Mathematics Subject Classification. Primary 35J10, 46B70.

(C)2006 American Mathematical Society Reverts to public domain 28 years from publication 
Here $L_{t}^{q} L_{x}^{r}$ denotes the space of functions $F(x, t)$ such that

$$
\|F\|_{L_{t}^{q} L_{x}^{r}}=\|\| F(\cdot, t)\left\|_{L^{r}\left(\mathbb{R}^{n}\right)}\right\|_{L^{q}(\mathbb{R})}<\infty,
$$

and the pairs $(q, r)$ that verify the conditions given in the Theorem 1.1 are called admissible pairs.

In this paper, we deal with the inhomogeneous part of the solution given in (3). The problem to solve is for which values of $q, r, \tilde{q}, \tilde{r} \in[1,+\infty]$ the following estimates hold:

$$
\begin{gathered}
\left\|\int_{0}^{t} e^{i(t-\tau) \Delta} F(\cdot, \tau) d \tau\right\|_{L_{t}^{q} L_{x}^{r}} \leq c\|F\|_{L_{t}^{\tilde{q}^{\prime}} L_{x}^{\tilde{r}^{\prime}}}, \\
\left\|\int_{-\infty}^{t} e^{i(t-\tau) \Delta} F(\cdot, \tau) d \tau\right\|_{L_{t}^{q} L_{x}^{r}} \leq c\|F\|_{L_{t}^{\tilde{q}^{\prime}} L_{x}^{\tilde{r}^{\prime}}} \\
\left\|\int_{-\infty}^{\infty} e^{i(t-\tau) \Delta} F(\cdot, \tau) d \tau\right\|_{L_{t}^{q} L_{x}^{r}} \leq c\|F\|_{L_{t}^{\tilde{q}^{\prime}} L_{x}^{\tilde{r}^{\prime}}} .
\end{gathered}
$$

The inequalities (5), (6) and (7) involve different operators, but all of them have similar properties. First of all, because of the scale, the exponents $q, r, \tilde{q}$ and $\tilde{r}$ have to verify the identity

$$
\frac{1}{\tilde{q}^{\prime}}-\frac{1}{q}+\frac{n}{2}\left(\frac{1}{\tilde{r}^{\prime}}-\frac{1}{r}\right)=1 .
$$

Observe that if $r$ and $\tilde{r}$ are fixed in (8) the difference $1 / \tilde{q}^{\prime}-1 / q$ is also fixed, so it is enough to give the values of $q$ for which the estimates hold. Therefore, we will represent the results in a plane, drawing on the $X$ axis the values of $1 / \tilde{r}^{\prime}$ and on the $Y$ axis the values of $1 / r$ (see Figure1), and we will say that the estimates are true at point $P$ for some values of $q$. On the other hand, if the estimates are true with a pair $(q, r)$ on the left and a pair $\left(\tilde{q}^{\prime}, \tilde{r}^{\prime}\right)$ on the right, then they must also be true when we switch the roles of $(q, r)$ and $(\tilde{q}, \tilde{r})$. Therefore, the estimates are true at point $P$ when $a<1 / q_{0}<b$, if and only if they are true at $Q$ when $1-b<1 / \tilde{q}_{0}^{\prime}<1-a$. So the picture must be symmetric with respect to the line called line of symmetry, and then it is enough to study what happens on the right-hand side of this line.

Although the inequalities (5), (6) and (7) have similar properties, they are not equivalent in general, but we do have that (6) implies (5) and (7). In order to prove that (6) implies (5), divide the norm in the variable $t$ given in (5) in two, for $t \geq 0$ and for $t<0$. When $t \geq 0$ we can write $[0, t)=(-\infty, t) \cap[0, \infty)$, and then apply (6). The case $t<0$ can be reduced to the case $t \geq 0$ by just making some changes of variable. On the other hand, splitting the integral in (7) into two integrals and making changes of variable, one can see that (6) also implies (7). A result of M. Christ and A. Kiselev asserts that (6) and (7) are equivalent when $\tilde{q}^{\prime}<q$ (see [4), but the case $q=\tilde{q}^{\prime}$ is the interesting one, because it corresponds to the borderline $\overline{A B}$ in Figure 2 .

The known results about the Strichartz estimates given in (5), (6) and (7) are due to several authors. In the inequality (2) we find the first result, which was proved by R. Strichartz in 1977, and it corresponds to the case $q=r=\tilde{q}=\tilde{r}=2(n+2) / n$. After that, J. Ginebre and G. Velo in 1985 (see [5]), K. Yajima in 1987 (see [15]), and T. Cazenave and F.B. Weissler in 1988 (see [2]) proved the estimates when $(q, r)$ 


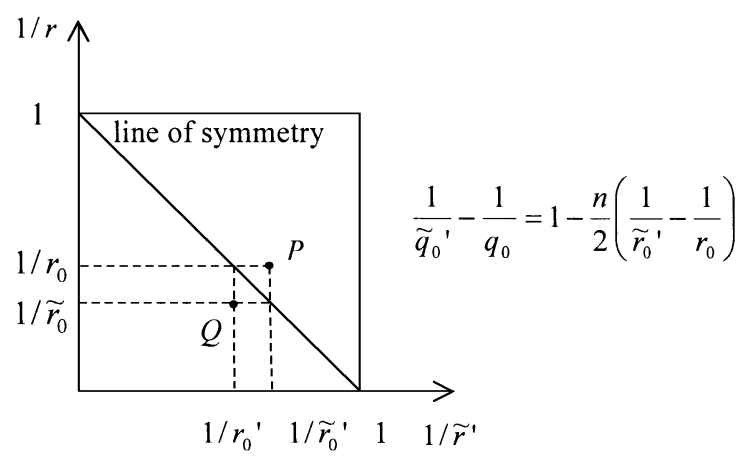

Figure 1. The Strichartz estimates given in (5), (6) and (7) are true at point $P$ with $q=q_{0}$ and $\tilde{q}^{\prime}=\tilde{q}_{0}^{\prime}$, if and only if they are true at point $Q$, with $q=\tilde{q}_{0}$ and $\tilde{q}^{\prime}=q_{0}^{\prime}$.

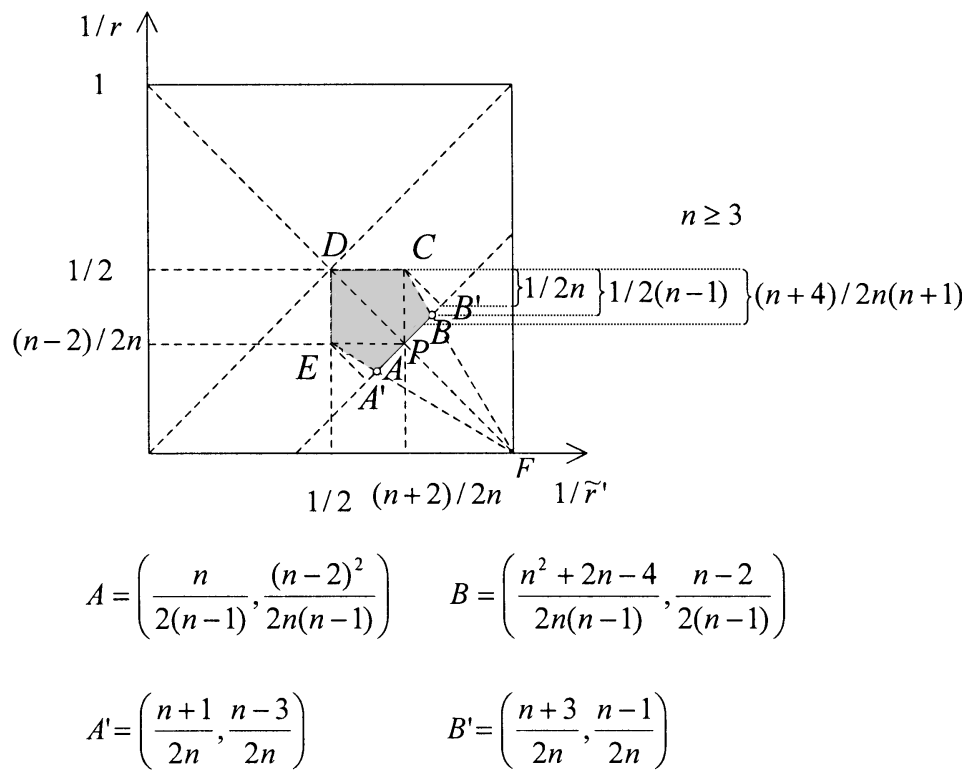

Figure 2. The Strichartz estimates given in (5), (6) and (7) are true in the pentagon with vertices $A, B, C, D$ and $E$ for certain values of $q$.

and $(\tilde{q}, \tilde{r})$ are admissible pairs with $q \neq 2$ and $\tilde{q} \neq 2$. In 1998, M. Keel and T. Tao (see [7) obtained the result for admissible pairs with $q=2$ or $\tilde{q}=2$. Note that for fixed $r$ and $\tilde{r}$, if we take $q$ and $\tilde{q}$ such that $(q, r)$ and $(\tilde{q}, \tilde{r})$ are admissible pairs the identity (8) holds, but there are other values of $q$ and $\tilde{q}$ verifying (8) for which the estimates (5), (6) and (7) could be true. The first result for pairs different from the admissible pairs is due to T. Cazenave and F. Weissler (see [3]). In 1992, they proved the estimates when $r=\tilde{r}$ with $2<r<2 n /(n-2)$ if $n \geq 3$ (open segment $\overline{D P}$ in Figure 2), $2<r<\infty$ if $n=2$, or $2<r \leq \infty$ if $n=1,1 / q<n(1 / 2-1 / r)$, and $q$ and $\tilde{q}$ verify (8), obtaining the estimates not only for admissible pairs $(q, r)$, 

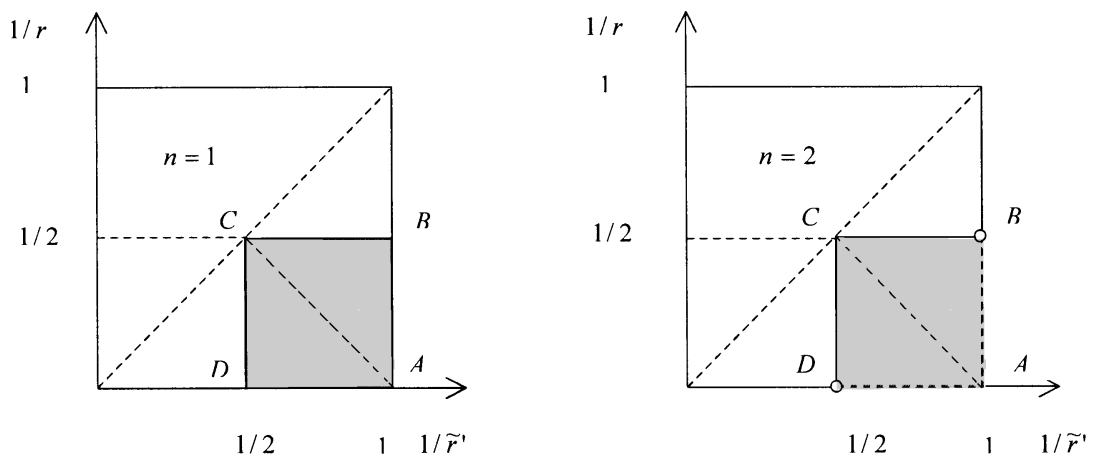

Figure 3. The Strichartz estimates given in (5), (6) and (7) are true inside the triangle $\widehat{A B C}$ for all possible $q$.

$(\tilde{q}, \tilde{r})$. Later, in 1994 T. Kato (see [6]) proved the estimates inside the square with vertices $C, D, E$ and $P$ when $1 / q<n(1 / 2-1 / r), 1 / \tilde{q}<n(1 / 2-1 / \tilde{r})$, and (8) is verified.

The proofs of J. Ginebre and G. Velo, and T. Cazenave and F. Weissler use the ideas of R. Strichartz. In this paper, we will follow the ideas of M. Keel and T. Tao to get some new results that we will prove in section 2, In dimension $n \geq 3$ (see Figure 21), we get that the estimates (5), (6) and (7) are true inside the triangle $\widehat{C D R}$ when $q=\infty$, obtaining the sharp result in this triangle. We also add to the known results the triangle $\widehat{B C P}$. More precisely, on the segment $\overline{B P}$ we have the estimates just for some values of $q$, for instance, we prove them at point $P$ when $2 n /(n+2) \leq q \leq 2 n /(n-2)$, improving the result of M. Keel and T. Tao, who obtained the estimates at point $P$ with $q=\tilde{q}=2$. The situation in the rest of the triangle is better, and we get the estimates for all possible $q$, except for $q=\infty$. The improvement we get in dimension $n=1$ or $n=2$ is just the critical case $q=\infty$ in the triangle $\widehat{A B C}$ (see Figure 3 ).

Concerning negative results, in section 3 we give some counterexamples which prove that the estimates (5), (6) and (7) are false outside the pentagon with vertices $A^{\prime}, B^{\prime}, C, D$ and $E$ for any $q$. However, we do not know what happens in either of the triangle $\widehat{B B^{\prime} C}$ or in its equivalent by duality $\widehat{A A^{\prime} E}$. In dimension $n=2$ we know that estimates (5) and (6) are false at point $A$ for any $q$. This is a trivial generalization of a result of $\mathrm{T}$. Tao given in [12, but the argument fails with estimate (77).

\section{Positive Results}

In this section we present two theorems. The first one is the main result of this paper, where we prove the estimates (5), (6) and (7) on the borderline $\overline{A B}$ (see Figure 2). In the second one we add to the known results the triangles $\widehat{B C P}$ and $\widehat{A E P}$.

Theorem 2.1. Let be $n \geq 3$ and $r, \tilde{r}$ such that

$$
\frac{1}{\tilde{r}^{\prime}}-\frac{1}{r}=\frac{2}{n}
$$


The Strichartz estimates given in (5), (6) and (7) hold when

$$
\begin{cases}\frac{1}{2(n-1)}<\frac{1}{2}-\frac{1}{r}<\frac{n+4}{2 n(n+1)}, & \text { if } \quad \frac{1}{r} \leq \frac{1}{q}=\frac{1}{\tilde{q}^{\prime}}<1-\frac{n}{2}\left(\frac{1}{\tilde{r}^{\prime}}+\frac{1}{r}-1\right), \\ \frac{n+4}{2 n(n+1)} \leq \frac{1}{2}-\frac{1}{r} \leq \frac{3}{2(n+1)}, & \text { if } \quad \frac{1}{r} \leq \frac{1}{q}=\frac{1}{\tilde{q}^{\prime}} \leq \frac{1}{\tilde{r}^{\prime}}, \\ \frac{3}{2(n+1)}<\frac{1}{2}-\frac{1}{r}<\frac{3 n-4}{2 n(n-1)}, & \text { if } \quad-\frac{n}{2}\left(\frac{1}{\tilde{r}^{\prime}}+\frac{1}{r}-1\right)<\frac{1}{q}=\frac{1}{\tilde{q}^{\prime}} \leq \frac{1}{\tilde{r}^{\prime}} .\end{cases}
$$

Proof. It is enough to prove estimate (6). We divide the proof into four steps.

Step 1. Following [7, we use the bilinear expression of (6), which is

$$
|T(F, G)| \leq c\|F\|_{L_{t}^{\tilde{q}^{\prime}} L_{x}^{\tilde{r}^{\prime}}}\|G\|_{L_{t}^{q^{\prime}} L_{x}^{r^{\prime}}},
$$

where $T(F, G)$ is the bilinear form

$$
T(F, G)=\int_{-\infty}^{+\infty} \int_{-\infty}^{t}\left\langle e^{-i \tau \Delta} F(\cdot, \tau), e^{-i t \Delta} G(\cdot, t)\right\rangle_{L_{x}^{2}} d \tau d t .
$$

Decomposing the operator $T$ dyadically, it is enough to prove that

$$
\sum_{j \in \mathbb{Z}}\left|T_{j}(F, G)\right| \leq c\|F\|_{L_{t}^{\tilde{q}^{\prime}} L_{x}^{\tilde{r}^{\prime}}}\|G\|_{L_{t}^{q^{\prime}} L_{x}^{r^{\prime}}}
$$

where for $j \in \mathbb{Z}$,

$$
T_{j}(F, G)=\int_{-\infty}^{+\infty} \int_{t-2^{j+1}<\tau \leq t-2^{j}}\left\langle e^{-i \tau \Delta} F(\cdot, \tau), e^{-i t \Delta} G(\cdot, t)\right\rangle_{L_{x}^{2}} d \tau d t .
$$

Step 2. In the next lemma we present a family of estimates for the operators $T_{j}$ that will allow us to add in (10).

Lemma 2.2. Let be $r$ and $\tilde{r}$ such that $2 \leq r, \tilde{r} \leq \infty$ and

$$
\frac{n-2}{n}\left(1-\frac{1}{\tilde{r}^{\prime}}\right) \leq \frac{1}{r} \leq \frac{n}{n-2}\left(1-\frac{1}{\tilde{r}^{\prime}}\right), \quad \text { if } n \geq 3
$$

(kite of vertices $C, D, E$ and $F$ in Figure 2). Then, for all $q$ and $\tilde{q}$ verifying

$$
0 \leq \frac{1}{q} \leq \frac{1}{\tilde{q}^{\prime}}<1-\frac{n}{2}\left(\frac{1}{\tilde{r}^{\prime}}+\frac{1}{r}-1\right), \quad \text { if } \quad \frac{1}{\tilde{r}^{\prime}}+\frac{1}{r} \geq 1,
$$

or

$$
-\frac{n}{2}\left(\frac{1}{\tilde{r}^{\prime}}+\frac{1}{r}-1\right)<\frac{1}{q} \leq \frac{1}{\tilde{q}^{\prime}} \leq 1, \quad \text { if } \quad \frac{1}{\tilde{r}^{\prime}}+\frac{1}{r} \leq 1,
$$

the following estimates hold for all $j \in \mathbb{Z}$ :

$$
\left|T_{j}(F, G)\right| \leq c 2^{-j \beta(\tilde{q}, q, \tilde{r}, r)}\|F\|_{L_{t}^{\tilde{q}^{\prime}} L_{x}^{\tilde{r}^{\prime}}}\|G\|_{L_{t}^{q^{\prime}} L_{x}^{r^{\prime}}},
$$

with $T_{j}$ the operator defined in (11) and

$$
\beta(\tilde{q}, q, \tilde{r}, r)=\left(\frac{1}{\tilde{q}^{\prime}}-\frac{1}{q}\right)+\frac{n}{2}\left(\frac{1}{\tilde{r}^{\prime}}-\frac{1}{r}\right)-1 .
$$


Observe that if we take $\tilde{q}, q, \tilde{r}$ and $r$ verifying (8), due to the scale of (15), (6) and (17), $\beta=0$, and then we cannot sum in (10) from inequalities (12). We solve this problem, for a kind of function, in the next step.

Step 3. Let us prove (10) for model functions $F_{k}$ and $G_{m}$ defined for $k, m \in \mathbb{Z}$ by

$$
F_{k}(x, \tau)=2^{-k / \tilde{r}^{\prime}} f(\tau) \chi_{I_{k}(\tau)}(x), \quad G_{m}(x, t)=2^{-m / r^{\prime}} g(t) \chi_{J_{m}(t)}(x),
$$

with $\left|I_{k}(\tau)\right|=2^{k} \forall \tau \in \mathbb{R}$ and $\left|J_{m}(t)\right|=2^{m} \forall t \in \mathbb{R}$.

Given a point $\left(1 / \tilde{r}^{\prime}, 1 / r\right)$ on the open segment $\overline{A B}$ (see Figure 21), and writing Lemma 2.2 for these model functions, with $\tilde{r}=a$ and $r=b$, we have that

$$
\left|T_{j}\left(F_{k}, G_{m}\right)\right| \leq c 2^{\left(k-j \frac{n}{2}\right)\left(\frac{1}{a^{\prime}}-\frac{1}{\vec{r}^{\prime}}\right)+\left(m-j \frac{n}{2}\right)\left(\frac{1}{r}-\frac{1}{b}\right)}\|f\|_{L_{t}^{\tilde{q}^{\prime}}}\|g\|_{L_{t}^{q^{\prime}}},
$$

for all $\left(1 / a^{\prime}, 1 / b\right)$ in a neighborhood of the point $\left(1 / \tilde{r}^{\prime}, 1 / r\right)$. Therefore, we can optimize in $a$ and $b$ to assert that there exists an $\varepsilon=\varepsilon(\tilde{r}, r)>0$ such that

$$
\left|T_{j}\left(F_{k}, G_{m}\right)\right| \leq c 2^{-\varepsilon\left(\left|k-j \frac{n}{2}\right|+\left|m-j \frac{n}{2}\right|\right)}\|f\|_{L_{t}^{\tilde{q}^{\prime}}}\|g\|_{L_{t}^{q^{\prime}}}
$$

These estimates allow us to sum in (10), concluding the proof for model functions.

Step 4. Finally, we deal with arbitrary functions, decomposing them into sums of model functions through the following lemma.

Lemma 2.3 (Atomic decomposition of $L^{p}$. See [10, e.g.). Let $1<p<\infty$. Then, any $f \in L^{p}$ can be written as

$$
f=\sum_{k \in \mathbb{Z}} c_{k} \chi_{k}
$$

where $\chi_{k}$ is a function bounded by $O\left(2^{-k / p}\right)$ and supported on a set of measure $O\left(2^{k}\right)$, and $c_{k}$ is a non-negative constant such that $\left\|c_{k}\right\|_{\ell^{p}} \leq c\|f\|_{L^{p}}$.

For fixed $\tau$ and $t, F(\cdot, \tau) \in L^{\tilde{r}^{\prime}}$ and $G(\cdot, t) \in L^{r^{\prime}}$, then, by the previous lemma,

$$
\begin{aligned}
F(x, \tau) & =\sum_{k \in \mathbb{Z}} F_{k}(x, \tau)=\sum_{k \in \mathbb{Z}} f_{k}(\tau) \tilde{\chi}_{k, \tau}(x) \quad \text { and } \\
G(x, t) & =\sum_{m \in \mathbb{Z}} G_{m}(x, t)=\sum_{m \in \mathbb{Z}} g_{m}(t) \chi_{m, t}(x), \quad \text { with }
\end{aligned}
$$

$\left|\tilde{\chi}_{k, \tau}\right|=O\left(2^{-k / \tilde{r}^{\prime}}\right),\left|\chi_{m, t}\right|=O\left(2^{-m / r^{\prime}}\right),\left|\operatorname{Supp} \tilde{\chi}_{k, \tau}\right|=O\left(2^{k}\right),\left|\operatorname{Supp} \chi_{m, t}\right|=O\left(2^{m}\right)$,

$$
\|\| f_{k}(\tau)\left\|_{\ell_{k}^{\tilde{r}^{\prime}}}\right\|_{L_{\tau}^{\tilde{q}^{\prime}}} \leq c\|F\|_{L_{\tau}^{\tilde{q}^{\prime}} L_{x}^{\tilde{r}^{\prime}}} \text { and }\|\| g_{k}(t)\left\|_{\ell_{k}^{r^{\prime}}}\right\|_{L_{t}^{q^{\prime}}} \leq c\|G\|_{L_{t}^{q^{\prime}} L_{x}^{r^{\prime}}},
$$

where Supp $f$ denotes the support of a function $f$. Then, by Step 3, we have that

$$
\begin{aligned}
\sum_{j \in \mathbb{Z}}\left|T_{j}(F, G)\right| & \leq c \sum_{j \in \mathbb{Z}} \sum_{k \in \mathbb{Z}} \sum_{m \in \mathbb{Z}}\left|T_{j}\left(F_{k}, G_{m}\right)\right| \\
& \leq c \sum_{k \in \mathbb{Z}} \sum_{m \in \mathbb{Z}}(1+|k-m|) 2^{-\varepsilon|k-m|}\left\|f_{k}\right\|_{L_{t}^{\tilde{q}^{\prime}}}\left\|g_{m}\right\|_{L_{t}^{q^{\prime}}} \\
& =c\|f(h * g)\|_{\ell^{1}}
\end{aligned}
$$

where $f(k)=\left\|f_{k}(t)\right\|_{L_{t}^{\tilde{q}^{\prime}}}, g(k)=\left\|g_{k}(t)\right\|_{L_{t}^{q^{\prime}}}$ and $h(k)=(1+|k|) 2^{-\varepsilon|k|}$. Taking $a$ and $b$ such that $1+1 / a=1 / b+1 / c$, with $1 \leq a, b, c \leq \infty$, and using Hölder's inequality and Young's inequality, we get that

$$
\sum_{j \in \mathbb{Z}}\left|T_{j}(F, G)\right| \leq c\|f\|_{\ell^{a^{\prime}}}\|g\|_{\ell^{b}}
$$


We finish proving that, by Minkowski's inequality and (15), $\|f\|_{\ell^{a^{\prime}}} \leq\|F\|_{L_{\tau}^{\tilde{q}^{\prime}} L_{x}^{\tilde{r}^{\prime}}}$ if $\tilde{q}^{\prime} \leq a^{\prime}$ and $\tilde{r}^{\prime} \leq a^{\prime}$. Similarly, $\|g\|_{\ell^{b}} \leq\|G\|_{L_{t}^{q^{\prime}} L_{x}^{r^{\prime}}}$ if $b^{\prime} \leq q$ and $b^{\prime} \leq r$.

Because of the conditions that $a$ and $b$ must verify, we have the restrictions $\tilde{r}^{\prime} \leq q$ and $\tilde{q}^{\prime} \leq r$. The rest of restrictions for $q$ or $\tilde{q}^{\prime}$ given on the theorem are sharp, as one can see from the identity (8) and Proposition 3.1 parts i) and ii).

Proof of Lemma 2.2. Because of the scale, it is enough to prove (12) when $j=0$; since $T_{0}$ is localized in time, if $\tilde{q}^{\prime} \leq q$, we may assume that $F$ and $G$ are supported on a time interval of length $O(1)$.

We get the result by interpolation of the following cases (see Figure 21):

i) $r=\tilde{r}=\infty$ and $1 \leq \tilde{q}^{\prime} \leq q \leq \infty \quad$ (point $F$ ),

ii) $r=\tilde{r}=2$ and $1 \leq \tilde{q}^{\prime} \leq q \leq \infty \quad($ point $D)$,

iii) $r=2,\left\{\begin{array}{ll}\tilde{r}=\infty \text { and } 2 \leq \tilde{q}^{\prime} \leq q \leq \infty, & \text { if } n=1, \\ 2 \leq \tilde{r}<\infty \text { and } 2<\tilde{q}^{\prime} \leq q \leq \infty, & \text { if } n=2, \\ \tilde{r}=2 n /(n-2) \text { and } 2 \leq \tilde{q}^{\prime} \leq q \leq \infty, & \text { if } n \geq 3\end{array} \quad\right.$ (point $\left.C\right)$.

iv) $\tilde{r}=2,\left\{\begin{array}{ll}r=\infty \text { and } 1 \leq \tilde{q}^{\prime} \leq q \leq 2, & \text { if } n=1, \\ 2 \leq r<\infty \text { and } 1 \leq \tilde{q}^{\prime} \leq q<2, & \text { if } n=2, \\ r=2 n /(n-2) \text { and } 1 \leq \tilde{q}^{\prime} \leq q \leq 2, & \text { if } n \geq 3\end{array}\right.$ (point $\left.E\right)$.

Writing the Schrödinger operator for $t \neq 0$ as a convolution operator with kernel $(4 \pi i t)^{-n / 2} e^{i|\cdot|^{2} / 4 t}$, it is clear that $\left\|e^{i t \Delta} f\right\|_{L^{\infty}\left(\mathbb{R}^{n}\right)} \leq c|t|^{-n / 2}\|f\|_{L^{1}\left(\mathbb{R}^{n}\right)}$. Therefore,

$$
\begin{aligned}
\left|T_{0}(F, G)\right| & =\left|\int_{-\infty}^{+\infty} \int_{t-2<\tau \leq t-1}\left\langle e^{i(t-\tau) \Delta} F(\cdot, \tau), G(\cdot, t)\right\rangle_{L_{x}^{2}} d \tau d t\right| \\
& \leq c \int_{-\infty}^{+\infty} \int_{t-2<\tau \leq t-1}|t-\tau|^{-n / 2}\|F(\cdot, \tau)\|_{L_{x}^{1}}\|G(\cdot, t)\|_{L_{x}^{1}} d \tau d t \\
& \leq c\|F\|_{L_{t}^{1} L_{x}}\|G\|_{L_{t}^{1} L_{x}^{1}},
\end{aligned}
$$

for all $q, \tilde{q} \geq 1$, by Hölder's inequality.

On the other hand, as the Schrödinger operator is an isometry in $L^{2}\left(\mathbb{R}^{n}\right)$, we have that

$$
\left|T_{0}(F, G)\right| \leq c\|F\|_{L_{t}^{1} L_{x}^{2}}\|G\|_{L_{t}^{1} L_{x}^{2}},
$$

and we get ii) by Hölder's inequality.

To prove iii) when $n \neq 2$, we use the Cauchy-Schwartz inequality, the dual of estimate (4) with $q=2$, and Hölder's inequality. For $n \geq 3$ we get that

$$
\begin{aligned}
\left|T_{0}(F, G)\right| & \leq \int_{-\infty}^{+\infty}\left\|\int_{t-2<\tau \leq t-1} e^{-i \tau \Delta} F(\cdot, \tau) d \tau\right\|_{L_{x}^{2}}\left\|e^{-i t \Delta} G(\cdot, t)\right\|_{L_{x}^{2}} d t \\
& \leq c \sup _{t \in \mathbb{R}}\left\|\chi_{(t-2, t-1]}(\tau) F(\cdot, \tau)\right\|_{L_{t}^{2} L_{x}^{2 n /(n+2)}}\|G\|_{L_{t}^{1} L_{x}^{2}} \\
& \leq c\|F\|_{L_{t}^{\tilde{q}^{\prime}} L_{x}^{2 n /(n+2)}}\|G\|_{L_{t}^{q^{\prime}} L_{x}^{2}},
\end{aligned}
$$

whenever $\tilde{q}^{\prime} \geq 2$ and $q \geq 1$. In the case $n=2$, (4) is false for $q=2$, but it is true for $q=2+\varepsilon$ with $\varepsilon>0$ as small as we want, and then we can proceed in the same way. A similar argument gives iv). 
We finish this section with a result for the triangle $\widehat{B C P}$ and its equivalent by duality $\widehat{A E P}$ (see Figure 2). The situation inside these triangles is better than the situation on the borderline $\overline{A B}$, and the result that we get is almost sharp in time, in the sense that it is formulated for all possible $q$ and $\tilde{q}$ except $q=\infty$ or $\tilde{q}=\infty$, as one can see from Proposition 3.1 parts i), ii) and iii).

Theorem 2.4. Let be $n \geq 3$. The Strichartz estimates given in (5), (6) and (7) hold when $\tilde{r}, r, \tilde{q}$ and $q$ verify the identity

$$
\frac{1}{\tilde{q}^{\prime}}-\frac{1}{q}+\frac{n}{2}\left(\frac{1}{\tilde{r}^{\prime}}-\frac{1}{r}\right)=1
$$

due to the scale, and one of the following conditions:

i) $\frac{1}{\tilde{r}^{\prime}} \geq \frac{n+2}{2 n}, \quad \frac{1}{\tilde{r}^{\prime}}-\frac{2}{n}<\frac{1}{r} \leq \frac{n}{n-2}\left(1-\frac{1}{\tilde{r}^{\prime}}\right) \quad$ (triangle $\left.\widehat{B C P}\right)$

$$
\text { and } 0<\frac{1}{q} \leq \frac{1}{\tilde{q}^{\prime}}<1-\frac{n}{2}\left(\frac{1}{\tilde{r}^{\prime}}+\frac{1}{r}-1\right)
$$

ii) $\frac{1}{r} \leq \frac{n-2}{2 n}, \quad 1-\left(\frac{n}{n-2}\right) \frac{1}{r} \leq \frac{1}{\tilde{r}^{\prime}}<\frac{1}{r}+\frac{2}{n} \quad$ (triangle $\left.\widehat{A E P}\right)$

$$
\text { and } \quad-\frac{n}{2}\left(\frac{1}{\tilde{r}^{\prime}}+\frac{1}{r}-1\right)<\frac{1}{q} \leq \frac{1}{\tilde{q}^{\prime}}<1
$$

The proof of this theorem is analogous to the proof of Theorem 2.1 except for steps three and four, which are replaced by a bilinear interpolation argument that we present in the next lemma, and that you can find in [1] (section 3.13, exercise $5(\mathrm{~b}))$.

Lemma 2.5. If $A_{0}, A_{1}, B_{0}, B_{1}, C_{0}$ and $C_{1}$ are Banach spaces, and $T$ is a bilinear operator such that

$$
\begin{aligned}
& T: A_{0} \times B_{0} \longrightarrow C_{0}, \\
& T: A_{0} \times B_{1} \longrightarrow C_{1}, \\
& T: A_{1} \times B_{0} \longrightarrow C_{1},
\end{aligned}
$$

then, whenever $0<\theta_{0}, \theta_{1}<\theta=\theta_{0}+\theta_{1}<1,1 \leq p, q, r \leq \infty$ and $1 \leq \frac{1}{p}+\frac{1}{q}$, we have that

$$
T:\left(A_{0}, A_{1}\right)_{\theta_{0}, p r} \times\left(B_{0}, B_{1}\right)_{\theta_{1}, q r} \longrightarrow\left(C_{0}, C_{1}\right)_{\theta, r} .
$$

Here $\left(C_{0}, C_{1}\right)_{\theta, r}$ denotes the Banach space obtained from $C_{0}$ and $C_{1}$ by the real interpolation method.

Proof of Theorem 2.4. Arguing as in Step 1 of the proof of Theorem 2.1, it is enough to prove the estimate (10), which can be written in the form

$$
\|\mathcal{T}\|_{\ell_{1}^{0}(\mathbb{C})} \leq c\|F\|_{L_{t}^{\tilde{q}^{\prime}} L_{x}^{\tilde{r}^{\prime}}}\|G\|_{L_{t}^{q^{\prime}} L_{x}^{r^{\prime}}}
$$

where $\mathcal{T}=\left\{T_{j}\right\}_{j \in \mathbb{Z}}$ is a vector-valued bilinear operator with $T_{j}$ given in (11).

In general, for $s \in \mathbb{R}$ and $1 \leq p \leq \infty, \ell_{p}^{s}(\mathbb{C})$ denotes the Banach space of sequences $\bar{x}=\left\{x_{k}\right\}_{k \in \mathbb{Z}}$ such that

$$
\|\bar{x}\|_{\ell_{p}^{s}}= \begin{cases}\left(\sum_{k \in \mathbb{Z}}\left(2^{k s}\left|x_{k}\right|\right)^{p}\right)^{1 / p}<\infty, & \text { if } p \neq \infty, \\ \sup _{k \in \mathbb{Z}}\left(2^{k s}\left|x_{k}\right|\right)<\infty, & \text { if } p=\infty .\end{cases}
$$


Fixed $\tilde{r}$ and $r$ under the conditions of the theorem, by Lemma 2.2, we can take certain $\tilde{q}_{0}, q_{0}, \tilde{q}_{1}$ and $q_{1}$ verifying the identity $\frac{1}{q_{0}}-\frac{1}{q_{1}}=\frac{1}{\tilde{q}_{1}^{\prime}}-\frac{1}{\tilde{q}_{0}^{\prime}}$ such that

$$
\begin{aligned}
& \mathcal{T}: L_{t}^{\tilde{q}_{0}^{\prime}} L_{x}^{\tilde{r}^{\prime}} \times L_{t}^{q_{0}^{\prime}} L_{x}^{r^{\prime}} \longrightarrow \ell_{\infty}^{\beta\left(\tilde{q}_{0}, q_{0}, \tilde{r}, r\right)}(\mathbb{C})=\ell_{\infty}^{\beta_{0}}(\mathbb{C}), \\
& \mathcal{T}: L_{t}^{\tilde{q}_{0}^{\prime}} L_{x}^{\tilde{r}^{\prime}} \times L_{t}^{q_{1}^{\prime}} L_{x}^{r^{\prime}} \longrightarrow \ell_{\infty}^{\beta\left(\tilde{q}_{0}, q_{1}, \tilde{r}, r\right)}(\mathbb{C})=\ell_{\infty}^{\beta_{1}}(\mathbb{C}), \\
& \mathcal{T}: L_{t}^{\tilde{q}_{1}^{\prime}} L_{x}^{\tilde{r}^{\prime}} \times L_{t}^{q_{0}^{\prime}} L_{x}^{r^{\prime}} \longrightarrow \ell_{\infty}^{\beta\left(\tilde{q}_{1}, q_{0}, \tilde{r}, r\right)}(\mathbb{C})=\ell_{\infty}^{\beta_{1}}(\mathbb{C}) .
\end{aligned}
$$

Therefore, we can apply Lemma 2.5 with $r=1$ to the operator $\mathcal{T}$, obtaining that

$$
\mathcal{T}:\left(L_{t}^{\tilde{q}_{0}^{\prime}} L_{x}^{\tilde{r}^{\prime}}, L_{t}^{\tilde{q}_{1}^{\prime}} L_{x}^{\tilde{r}^{\prime}}\right)_{\theta_{0}, \tilde{q}^{\prime}} \times\left(L_{t}^{q_{0}^{\prime}} L_{x}^{r^{\prime}}, L_{t}^{q_{1}^{\prime}} L_{x}^{r^{\prime}}\right)_{\theta_{1}, q^{\prime}} \rightarrow\left(\ell_{\infty}^{\beta_{0}}(\mathbb{C}), \ell_{\infty}^{\beta_{1}}(\mathbb{C})\right)_{\theta, 1},
$$

whenever $0<\theta_{0}, \theta_{1}<\theta=\theta_{0}+\theta_{1}<1$ and $1 \leq \tilde{q}^{\prime} \leq q \leq \infty$. The proof follows using the next real interpolation space identities (see [1], Theorems 5.6.1 and 5.2.1):

i) $\left(\ell_{\infty}^{\beta_{0}}(\mathbb{C}), \ell_{\infty}^{\beta_{1}}(\mathbb{C})\right)_{\theta, 1}=\ell_{1}^{0}(\mathbb{C})$ if $q_{0} \neq q_{1}$ and $(1-\theta) \beta_{0}+\theta \beta_{1}=0$.

ii) $\left(L_{t}^{\tilde{q}_{0}^{\prime}} L_{x}^{\tilde{r}^{\prime}}, L_{t}^{\tilde{q}_{1}^{\prime}} L_{x}^{\tilde{r}^{\prime}}\right)_{\theta_{0}, \tilde{q}^{\prime}}=L_{t}^{\tilde{q}^{\prime}} L_{x}^{\tilde{r}^{\prime}}$ and $\left(L_{t}^{q_{0}^{\prime}} L_{x}^{r^{\prime}}, L_{t}^{q_{1}^{\prime}} L_{x}^{r^{\prime}}\right)_{\theta_{1}, q^{\prime}}=L_{t}^{q^{\prime}} L_{x}^{r^{\prime}}$, if

$$
\frac{1}{\tilde{q}^{\prime}}=\frac{\left(1-\theta_{0}\right)}{\tilde{q}_{0}^{\prime}}+\frac{\theta_{0}}{\tilde{q}_{1}^{\prime}} \text { and } \frac{1}{q}=\frac{\left(1-\theta_{1}\right)}{q_{0}}+\frac{\theta_{1}}{q_{1}},
$$

respectively.

Remark 2.6. In the previous proof, for $r, \tilde{r}, q$ and $\tilde{q}$ fixed under the conditions of Theorem 2.4, it is essential to have the family of estimates given by Lemma 2.2 in a neighbourghood of the point $\left(1 / \tilde{q}^{\prime}, 1 / q\right)$. That is the reason why we do not get the result for the critical cases $q=\infty$ or $\tilde{q}=\infty$.

Remark 2.7. Theorem 2.4 holds even when $\left(1 / \tilde{r}^{\prime}, 1 / r\right)$ is inside the pentagon with vertices $A, B, C, D$ and $E$, but the result given is unknown just in the triangles $\widehat{B C P}$ and $\widehat{A E P}$.

Remark 2.8. In this paper we study Strichartz estimates for the Schrödinger equation, but similar results can be obtained for more general equations, where the Laplacian is replaced by the operator $\Delta_{x}^{a / 2}$ with $a>0$, which is defined by

$$
\left(\Delta_{x}^{a / 2} f\right)^{\uparrow}(\xi)=\left(-4 \pi^{2}|\xi|^{2}\right)^{a / 2} .
$$

\section{Negative Results}

In this section we give some counterexamples which show that the Strichartz estimates given in (5), (6) and (7) are false for some exponents.

Proposition 3.1. The estimates given in (5), (6) and (17) are false in the following cases:

i) $r<2$, or, $r=2$ and $q \neq \infty$, or, $r>2$ and $\frac{1}{q} \geq n\left(\frac{1}{2}-\frac{1}{r}\right)$,

ii) $\tilde{r}^{\prime}>2$, or, $\tilde{r}^{\prime}=2$ and $\tilde{q} \neq \infty$, or, $\tilde{r}^{\prime}<2$ and $\frac{1}{\tilde{q}^{\prime}} \leq 1-n\left(\frac{1}{\tilde{r}^{\prime}}-\frac{1}{2}\right)$,

iii) $q<\tilde{q}^{\prime}$

iv) $\frac{1}{\tilde{r}^{\prime}}-\frac{1}{r}>\frac{2}{n}$

v) $\frac{1}{\tilde{r}^{\prime}}+\frac{1}{r}>1+\frac{1}{n}$

vi) $\frac{1}{\tilde{r}^{\prime}}+\frac{1}{r}<1-\frac{1}{n}$.

Proof. By duality, i) is equivalent to ii) and v) is equivalent to vi). On the other hand, (6) implies (5) and (7), so it is enough to give counterexamples for (5) and (7). 
The counterexample for (7) in case i) is a function $F(x, t)$ with Fourier transform in both variables given by $\widehat{F}^{x, t}(\xi, \tau)=\varphi(\tau) e^{-\pi|\xi|^{2}}$, where $\varphi \in C^{\infty}(\mathbb{R})$ is supported in $(-4 \pi, 4 \pi), 0 \leq \varphi \leq 1$ and $\varphi(r)=1$ in $(-2 \pi, 2 \pi)$. If we denote the operator in (77) by $T T^{*}$, and starting from the expression of this operator via the Fourier transform, we have that

$$
\begin{aligned}
T T^{*} F(x, t) & =\int_{\mathbb{R}^{n}} e^{-4 \pi^{2} i t|\xi|^{2}} \varphi\left(-2 \pi|\xi|^{2}\right) e^{-\pi|\xi|^{2}} e^{2 \pi i x \cdot \xi} d \xi \\
& =(1+4 \pi i t)^{-\frac{n}{2}} e^{\frac{-\pi|x|^{2}}{1+4 \pi i t}}+I
\end{aligned}
$$

with

$$
I=\int_{\mathbb{R}^{n}} e^{-\pi|\xi|^{2}(1+4 \pi i t)}\left[\varphi\left(-2 \pi|\xi|^{2}\right)-1\right] e^{2 \pi i x \cdot \xi} d \xi
$$

Writing $I$ in polar coordinates and using the behaviour of $\widehat{d \sigma}$ at infinity, where $d \sigma$ is the measure over the unit sphere, it is easy to see that there exists $a>1$ such that if $t>a$ and $t<|x|<2 t$, then $|I| \leq c|t|^{-(n+1) / 2}$. Therefore, from (16) we have that $\left|T T^{*} F(x, t)\right| \geq c t^{-n / 2}$, and the result follows from here. For estimate (5), just replace $\varphi$ by a function $\psi \in C^{\infty}(\mathbb{R})$ supported in $(1 / 2,3 / 2)$ with $0 \leq \psi \leq 1$ and $\psi(1)=1$, and everything works.

In case iii) take as a counterexample the function

$$
H(x, t)=\sum_{j=0}^{N} H_{j}(x, t)=\sum_{j=0}^{N} F\left(x, t-t_{j}\right),
$$

with $F$ defined as in case i) and $t_{j}=j N$ for $j=0,1, \ldots, N$. Taking $N$ large enough, we have that $\|H\|_{L_{t}^{\tilde{q}^{\prime}} L_{x}^{\tilde{r}^{\prime}}} \leq c N^{1 / \tilde{q}^{\prime}}$. On the other hand, let

$$
B=\bigcup_{k=1}^{N-1} B_{k} \quad \text { with } \quad B_{k}=\left\{t / 1<\left|t-t_{k}\right|<2\right\} .
$$

The result follows because for $N$ large enough, we can write that

$$
\begin{aligned}
\left\|T T^{*} H\right\|_{L_{t}^{q} L_{x}^{r}}^{q} & \geq \sum_{k=1}^{N-1}\left\|T T^{*} H\right\|_{L^{q}\left(B_{k}\right) L_{x}^{r}}^{q} \\
& \geq c \sum_{k=1}^{N-1}\left(\left\|T T^{*} H_{k}\right\|_{L^{q}\left(B_{k}\right) L_{x}^{r}}-\left\|\sum_{j \neq k} T T^{*} H_{j}\right\|_{L^{q}\left(B_{k}\right) L_{x}^{r}}\right)^{q} \\
& \geq c \sum_{k=0}^{N}\left(1-N^{-\frac{n}{2}} \sum_{j \neq k}|j-k|^{-\frac{n}{2}}\right)^{q} \geq c N .
\end{aligned}
$$

Case iv) is consequence of case iii) and the identity (8), due to the scale.

Finally, in case $v$ ) the counterexample for (7) is a function $G(x, t)$ with Fourier transform given by $\widehat{G}^{x, t}(\xi, \tau)=\phi(|\xi| / 2) \phi(M(\tau+2 \pi))$, where $\phi \in C^{\infty}(\mathbb{R})$ is an even function supported in $(-1,1), 0 \leq \phi \leq 1$, and $M$ is a constant as large as we need. Using polar coordinates and the behaviour of $\widehat{d \sigma}$ at infinity, we have that if $|x|$ is large enough, then $\left|T T^{*} G(x, t)\right|=2|x|^{-(n-1) / 2}|I(x, t)|$, with

$$
I(x, t)=\int_{0}^{+\infty} r^{\frac{n-1}{2}} \phi\left(\frac{r}{2}\right) \phi\left(2 \pi M\left(r^{2}-1\right)\right) e^{-4 \pi^{2} i t r^{2}} \cos \left(2 \pi r|x|-(n-1) \frac{\pi}{4}\right) d r .
$$


Taking $M$ large enough, if $0<t<M / 2$ and $x \in A=\bigcup_{3 M / 2<m<2 M} A_{m}$ with

$$
A_{m}=\left\{x / \frac{m+\frac{n}{8}-\frac{1}{24}}{\left(1-\frac{M^{-1}}{2 \pi}\right)^{1 / 2}}<|x|<\frac{m+\frac{n}{8}+\frac{1}{24}}{\left(1+\frac{M^{-1}}{2 \pi}\right)^{1 / 2}}\right\} \quad(m \in \mathbb{N}),
$$

then $|I(x, t)| \geq c M^{-1}$ and therefore

$$
\left\|T T^{*} G\right\|_{L_{t}^{q} L_{x}^{r}} \geq\left\|T T^{*} G\right\|_{L_{t}^{q}(0, M / 2) L_{x}^{r}(A)} \geq c M^{-\frac{(n+1)}{2}+\frac{1}{q}+\frac{n}{r}} .
$$

The result follows making $M$ go to infinity, using the identity (8) and the fact that

$$
\|G\|_{L_{t}^{\tilde{q}^{\prime}} L_{x}^{\tilde{r}^{\prime}}} \leq c M^{-1+\frac{1}{\tilde{q}^{\prime}}} .
$$

For estimate (5), we take $\widehat{G}^{x, t}(\xi, \tau)=\psi(|\xi|) \phi(M(\tau+2 \pi))$, with $\psi, \phi$ as defined before, and we denote the operator by $\mathcal{U}$. Using the Fourier transform we write

$$
\mathcal{U} G(x, t)=\frac{-1}{2 \pi} \int_{\mathbb{R} \times \mathbb{R}^{n}} \frac{1-e^{-2 \pi i t\left(\tau+2 \pi|\xi|^{2}\right)}}{\tau+2 \pi|\xi|^{2}} \psi(|\xi|) \phi(M(\tau+2 \pi)) e^{2 \pi i t \tau+2 \pi i x \cdot \xi} d \tau d \xi .
$$

With a change of variable we have that

$$
\mathcal{U} G(x, t)=\frac{-1}{2 \pi} \int_{\mathbb{R}} I(\tau) \frac{\phi(M(\tau+2 \pi))}{|\tau|}\left(\sqrt{\frac{|\tau|}{2 \pi}}\right)^{n} e^{2 \pi i t \tau} d \tau
$$

where

$$
I(\tau)=\int_{\mathbb{R}^{n}} \frac{1-e^{-2 \pi i t|\tau|\left(|\xi|^{2}-1\right)}}{|\xi|^{2}-1} \psi\left(\sqrt{\frac{|\tau|}{2 \pi}}|\xi|\right) e^{2 \pi i x \cdot \sqrt{\frac{|\tau|}{2 \pi}} \xi} d \xi .
$$

Using polar coordinates we write

$$
I(\tau)=\int_{0}^{\infty} r^{n-1} \frac{1-e^{-2 \pi i t|\tau|\left(r^{2}-1\right)}}{r^{2}-1} \psi\left(\sqrt{\frac{|\tau|}{2 \pi}} r\right) \widehat{d \sigma}\left(\sqrt{\frac{|\tau|}{2 \pi}} r x\right) d r .
$$

In order to estimate $I(\tau)$ we need the complete asymptotic expansion of $d \sigma$, that is,

$$
\widehat{d \sigma}(\xi)=|\xi|^{-(n-1) / 2}\left(e^{2 \pi i|\xi|} \sum_{j=0}^{\infty} c_{j}|\xi|^{-j}+e^{-2 \pi i|\xi|} \sum_{j=0}^{\infty} \bar{c}_{j}|\xi|^{-j}\right), \quad \text { if }|\xi| \rightarrow \infty,
$$

with the coefficient $c_{j}$ given by

$$
c_{j}= \begin{cases}(-1)^{\frac{j}{2}} e^{-i \frac{(n-1) \pi}{4}} a_{j}, & \text { if } j \text { is even, } \\ (-1)^{\frac{j+1}{2}} e^{-i \frac{(n+1) \pi}{4}} a_{j}, & \text { if } j \text { is odd, }\end{cases}
$$

where $a_{j}=\frac{(4 \pi)^{-j} \Gamma\left(\frac{n-1}{2}+j\right)}{j ! \Gamma\left(\frac{n-1}{2}-j\right)}$ and $\Gamma$ denotes the Gamma function (see [10]). If $|x|$ is large enough we can use this expansion to write

$$
I(\tau)=|x|^{-\frac{(n-1)}{2}}\left(\sqrt{\frac{|\tau|}{2 \pi}}\right)^{-\frac{(n-1)}{2}} \sum_{j=0}^{\infty}|x|^{-j}\left(\sqrt{\frac{|\tau|}{2 \pi}}\right)^{-j}\left(c_{j} I_{j}^{1}(\tau)+\bar{c}_{j} I_{j}^{2}(\tau)\right),
$$


where

$$
\begin{aligned}
I_{j}^{1}(\tau) & =\int_{-\infty}^{+\infty} \frac{1-e^{-2 \pi i t|\tau|\left(r^{2}-1\right)}}{r-1} g_{j}(r) e^{2 \pi i \sqrt{\frac{|\tau|}{2 \pi}} r|x|} d r \\
I_{j}^{2}(\tau) & =\int_{-\infty}^{+\infty} \frac{1-e^{-2 \pi i t|\tau|\left(r^{2}-1\right)}}{r-1} g_{j}(r) e^{-2 \pi i \sqrt{\frac{|\tau|}{2 \pi}} r|x|} d r
\end{aligned}
$$

with

$$
g_{j}(r)=\frac{|r|^{\frac{n-1}{2}-j} \psi\left(\sqrt{\frac{|\tau|}{2 \pi}} r\right)}{r+1} .
$$

Now we write $I_{j}^{1}$ as the following sum:

$$
I_{j}^{1}(\tau)=I_{j}^{1, a}(\tau)+g_{j}(1)\left(I^{1, b}(\tau)-I^{1, c}(\tau)\right)
$$

with

$$
\begin{aligned}
I_{j}^{1, a}(\tau) & =\int_{-\infty}^{+\infty} \frac{1-e^{-2 \pi i t|\tau|\left(r^{2}-1\right)}}{r-1}\left(g_{j}(r)-g_{j}(1)\right) e^{2 \pi i \sqrt{\frac{|\tau|}{2 \pi}} r|x|} d r \\
I^{1, b}(\tau) & =p \cdot v \cdot \int_{-\infty}^{+\infty} \frac{e^{2 \pi i \sqrt{\frac{|\tau|}{2 \pi}} r|x|}}{r-1} d r \\
I^{1, c}(\tau) & =p \cdot v \cdot \int_{-\infty}^{+\infty} \frac{e^{-2 \pi i t|\tau|\left(r^{2}-1\right)}}{r-1} e^{2 \pi i \sqrt{\frac{|\tau|}{2 \pi}} r|x|} d r,
\end{aligned}
$$

where p.v. denotes the principal value.

First of all, using the mean value theorem and integrating by parts we obtain, as long as $M(\tau+2 \pi) \in \operatorname{Supp} \phi$, that

$$
\left|I_{j}^{1, a}(\tau)\right| \leq c M^{-1}, \quad \text { if } \quad \frac{M}{12}<t<\frac{M}{6}, \quad \frac{M}{16}<|x|<\frac{M}{8} .
$$

On the other hand,

$$
I^{1, b}(\tau)=i \pi e^{2 \pi i \sqrt{\frac{|\tau|}{2 \pi}}|x|} \operatorname{sgn}\left(\sqrt{\frac{|\tau|}{2 \pi}}|x|\right)=i \pi e^{2 \pi i \sqrt{\frac{\mid \tau \tau}{2 \pi}}|x|} .
$$

Finally, if we put $\mu=\frac{|x|}{\sqrt{t}}-2 \sqrt{t}|\tau| \sqrt{\frac{2 \pi}{\tau \mid}}$, making a change of variable we can write

$$
\begin{aligned}
I^{1, c}(\tau) & =e^{2 \pi i \sqrt{\frac{|\tau|}{2 \pi}}|x|} e^{i \frac{\mu^{2}}{4}} p \cdot v \cdot \int_{-\infty}^{+\infty} e^{-i \frac{(\mu-r)^{2}}{4}} \frac{d r}{r} \\
& =2 \sqrt{\pi} e^{2 \pi i \sqrt{\frac{\mid \tau \tau}{2 \pi}}|x|} e^{i \frac{\mu^{2}}{4}} e^{-i \frac{\pi}{4}} e^{-i \Delta}\left(p \cdot v \cdot \frac{1}{r}\right)(\mu) \\
& =\sqrt{\pi} e^{i \frac{\pi}{4}} e^{2 \pi i \sqrt{\frac{|\tau|}{2 \pi}}|x|}\left(2 \sqrt{\pi} e^{i \frac{\pi}{4}}-\int_{-\infty}^{\mu} e^{i \frac{y^{2}}{4}} d y\right) .
\end{aligned}
$$

Integrating by parts we get that

$$
I^{1, c}(\tau)=2 \pi i e^{2 \pi i \sqrt{\frac{|\tau|}{2 \pi}}|x|}+O\left(M^{-1 / 2}\right),
$$

for the same $\tau, t$, and $x$ for which we obtained (21). Using (21), (22) and (23) in (20) we have that

$$
I_{j}^{1}(\tau)=-i \frac{\pi}{2} \psi\left(\sqrt{\frac{|\tau|}{2 \pi}}\right) e^{2 \pi i \sqrt{\frac{|\tau|}{2 \pi}}|x|}+O\left(M^{-1 / 2}\right),
$$


if $M / 12<t<M / 6, M / 16<|x|<M / 8$ and $M(\tau+2 \pi) \in \operatorname{Supp} \phi$. In a similar way and under the same conditions, you can prove that

$$
I_{j}^{2}(\tau)=-i \frac{\pi}{2} \psi\left(\sqrt{\frac{|\tau|}{2 \pi}}\right) e^{-2 \pi i \sqrt{\frac{\sqrt{2 \pi \mid}}{2 \pi}}|x|}+O\left(M^{-1 / 2}\right) .
$$

With these two estimates, from (19) we have for $t$ and $x$ such that $M / 12<t<M / 6$ and $M / 16<|x|<M / 8$ with $M$ large enough, that

$$
I(\tau)=-i \pi\left(|x| \sqrt{\frac{|\tau|}{2 \pi}}\right)^{-\frac{(n-1)}{2}} \psi\left(\sqrt{\frac{|\tau|}{2 \pi}}\right) \cos \left(|x| \sqrt{2 \pi|\tau|}-(n-1) \frac{\pi}{4}\right)+O\left(M^{-\frac{n}{2}}\right),
$$

and therefore, from (18) we obtain that

$$
\begin{aligned}
& |\mathcal{U} G(x, t)| \\
& \quad \geq c M^{-\frac{(n-1)}{2}} \mid \int_{\mathbb{R}} \phi\left(M(\tau+2 \pi) \cos \left(|x| \sqrt{2 \pi|\tau|}-(n-1) \frac{\pi}{4}\right) \cos (2 \pi t \tau) d \tau \mid\right. \\
& \quad+O\left(M^{-\frac{(n+2)}{2}}\right) .
\end{aligned}
$$

From here, if we take $x \in A=\bigcup_{\frac{M}{15}<m<\frac{M}{10}} A_{m}$ and $t \in B=\bigcup_{\frac{\pi}{5}} \bigcup_{M<k<\frac{\pi}{3} M} B_{k}$, with $A_{m}$ defined in (17) and

$$
B_{k}=\left\{t / \frac{k-\frac{1}{6}}{2 \pi-M^{-1}}<t<\frac{k+\frac{1}{6}}{2 \pi+M^{-1}}\right\} \quad(k \in \mathbb{N}),
$$

we have that $|\mathcal{U} G(x, t)| \geq O\left(M^{-(n+1) / 2}\right)$ and therefore,

$$
\|\mathcal{U} F\|_{L_{t}^{q} L_{x}^{r}} \geq\|\mathcal{U} F\|_{L^{q}(B) L^{r}(A)} \geq c M^{-\frac{(n+1)}{2}+\frac{1}{q}+\frac{n}{r}} .
$$

We finish the proof as we did for the operator $T T^{*}$.

\section{ACKNOWLEDGMENTS}

I would like to thank Luis Vega for suggesting this problem to me and for his patient help, and to M. Keel and T. Tao for their enlightening conversations.

Note. After this paper was submitted, I learned of a preprint by D. Foschi where related results were obtained.

\section{REFERENCES}

1. J. Bergh and J.Löfström, Interpolation Spaces. An Introduction, Springer-Verlag, Berlin, Heidelberg, New York, 1976. MR0482275 (58:2349)

2. T. Cazenave and F.B. Weissler, The Cauchy problem for the nonlinear Schrödinger equation in $H^{1}$, Manuscripta Math. 61 (1988), 477-494. MR0952091 (89j:35114)

3. T. Cazenave and F.B. Weissler, Rapidly decaying solutions of the nonlinear Schrödinger equation, Commun. Math. Phys. 147 (1992), 75-100. MR1171761 (93d:35150)

4. M. Christ and A. Kiselev, Maximal functions associated to filtrations, Journ. Func. Anal. 179 (2001), 409-425. MR.1809116 (2001i:47054)

5. J. Ginibre and G. Velo, The global Cauchy problem for the nonlinear Schrödinger equation revisited, Ann. Inst. H. Poincaré Anal. Non Linéare 2 (1985), 309-327. MR0801582 (87b:35150)

6. T. Kato, An $L^{q, r}$-theory for nonlinear Schrödinger equations, Spectral and scattering theory and applications, Adv. Stud. Pure Math., vol. 23, Math. Soc. Japan, Tokyo, 1994, pp. 223-238. MR 1275405 (95i:35276)

7. M. Keel and T. Tao, Endpoint Strichartz estimates, Amer. J. Math. 120 (1998), 955-980. MR:1646048 (2000d:35018) 
8. S.J. Montgomery-Smith, Time decay for the bounded mean oscillation of solutions of the Schrödinger and wave equation, Duke Math. J. 91 (1998), 393-408. MR1600602 (99e:35006)

9. I.E. Segal, Space-time decay for solutions of wave equations, Adv. Math. 22 (1976), 304-311. MR 0492892(58:11945)

10. E.M. Stein, Harmonic Analysis. Real-variable Methods, Orthogonality, and Oscillatory Integrals, Princeton University Press, Princeton, New Jersey, 1993. MR1232192 (95c:42002)

11. R.S. Strichartz, Restrictions of Fourier transforms to quadratic surfaces and decay of solutions of wave equations, Duke Math. J. 44 (1977), 705-714. MR0512086 (58:23577)

12. T. Tao, Spherically averaged endpoint Strichartz estimates for the two-dimensional Schrödinger equation, Comm. Partial Differential Equations 25 (2000), 1471-1485. MR:1765155 (2001h:35038)

13. P.A. Tomas, A restriction theorem for the Fourier transform, Bull. Amer. Math. Soc. 81 (1975), 477-478. MR0358216 (50:10681)

14. P.A. Tomas, Restriction theorems for the Fourier transform, Harmonic Analysis in Euclidean Spaces (2 volumes), G. Weiss and W. Wainger (eds.), Proc. Sympos. Pure Math. \# 35, Amer. Math. Society (1979), part 1, 111-114. MR0545245 (81d:42029)

15. K. Yajima, Existence of solutions for Schrödinger evolution equations, Comm. Math. Phys. 110 (1987), 415-426. MR0891945 (88e:35048)

Departamento de Matemática Aplicada, Escuela Universitaria de Informática, Campus de Segovia - Universidad de Valladolid, Plaza de Santa Eulalia 9 y 11, 40005 Segovia, Spain

E-mail address: maricruz@eis.uva.es 